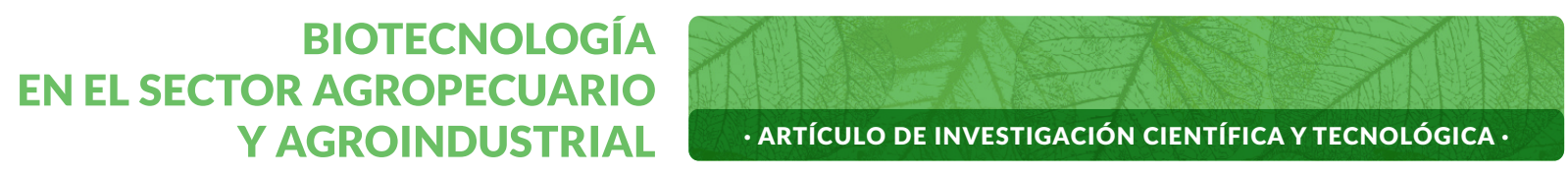

Vol. 18 No 2 • Julio - Diciembre 2020 • ISSN - 1692-3561 · ISSN-e 1909-9959 · DOI: http://dx.doi.org/10.18684

\title{
Efecto del periodo de recuperación en la producción y calidad nutricional de algunas especies forrajeras*
}

\section{Effect of the recovery period on the production and nutritional quality of some forage species}

\section{Efeito do período de recuperação na produção e qualidade nutricional de algumas espécies forrageiras}

\author{
DANIA FONSECA-LÓPEZ , ISRAEL ALBERTO BOHÓRQUEZ-MASMELA², \\ CARLOS EDUARDO RODRÍGUEZ-MOLANO ${ }^{3}$, NELSON JOSÉ VIVAS-QUILA ${ }^{4}$
}

Historial del Artículo

Recibido para evaluación: 12 de Septiembre 2019.

Aprobado para publicación: 4 de Mayo 2020.

* Proyecto de investigación de orígen: "Evaluación de la respuesta agronómica de algunas especies forrajeras de trópico alto como estrategia hacia una ganadería ecoeficiente". Financiación: Universidad Pedagógica y Tecnológica de Colombia, VIE convocatoria 781 de 2017 SGI 2405. Culminación: Fecha de finalización: 15 de junio de 2019.

1 Universidad Pedagógica y Tecnológica de Colombia. Facultad de Ciencias Agropecuarias, Grupo de Investigación en Bioquímica Y Nutrición Animal (GIBNA). Investigador, Magister en Ciencias Veterinarias. Tunja, Colombia. http://orcid.org/0000-0003-3877-3730

2 Universidad Pedagógica y Tecnológica de Colombia. Facultad de Ciencias Agropecuarias, Grupo de Investigación en Bioquímica Y Nutrición Animal (GIBNA). Profesor titular, Zootecnista. Tunja, Colombia. https://orcid.org/0000-0003-3905-1449

3 Universidad Pedagógica y Tecnológica de Colombia. Facultad de Ciencias Agropecuarias, Grupo de Investigación en Bioquímica Y Nutrición Animal (GIBNA). Profesor titular, Magister en Ciencias Biológicas. Tunja, Colombia. http://orcid.org/0000-0002-0862-3478

4 Universidad del Cauca, Facultad de Ciencias Agrarias, Grupo de Investigación Nutrición Agropecuaria. Profesor Titular, Doctor en Ciencias Agrarias. Popayán, Colombia. https:// orcid.org/0000-0003-0165-2863 


\section{RESUMEN}

La capacidad que tienen los forrajes para recuperarse del corte permite diseñar estrategias de manejo, por ello, se evaluaron diferentes tiempos de recuperación en temporada seca y de lluvias en Vicia sativa L., Avena sativa L., Trifolium repens L., Trifolium pratense L., Medicago sativa L., y Dactylis glomerata L. en Boyacá (2.700 msnm) en un diseño completamente al azar, con un arreglo factorial $4 \times 6$ que correspondió a cuatro periodos de recuperación (25, 30, 35 y 40 días) y seis forrajes. Las variables evaluadas fueron producción de materia seca (MS), altura, calidad nutricional, y disponibilidad de $\mathrm{N}$, Ca y $\mathrm{P}$ en el suelo al iniciar y al finalizar el ensayo. Los datos fueron analizados con Anova y la prueba de Tukey $(p<0,05)$. Se observó que la producción de forraje (MS) difirió en todas las especies entre los periodos de recuperación y el contenido proteico únicamente en avena, las demás variables no cambiaron estadísticamente. Se concluye que el tiempo óptimo de corte es de 40 días para trébol rojo, vicia y avena; de 35 días para trébol blanco y 30 días para pasto azul y alfalfa.

\section{ABSTRACT}

The ability of the forages to recover from being cut, allows to design management strategies, therefore different recovery periods in the dry and rainy season were evaluated in Vicia sativa L., Avena sativa L., Trifolium repens L., Trifolium pratense L., Medicago sativa L., y Dactylis glomerata L. in Boyacá (2.700 masl) in a completely random design, with a $4 \times 6$ factorial arrangement that corresponded to four recovery periods $(25,30,35$ and 40 days) and six forages. The variables evaluated were production of dry matter (MS), height, nutritional quality, and availability of $\mathrm{N}, \mathrm{Ca}$ and $\mathrm{P}$ in the soil at the beginning and end of the test. The data was analyzed with Anova and the Tukey test $(P 0,05)$. It was observed that forage production (DM) differed in all species between recovery periods and protein content only in forage oats, the other variables did not change statistically. It is concluded that the optimal cutting time is 40 days for red clover, Vicia and oats, 35 days for white clover and 30 days for cocksfoot and alfalfa.

\section{RESUMO}

A capacidade que têm as forragens para recuperar do corte permite desenhar estratégias de manejo, por isso se avaliaram diferentes períodos de recuperação na estação seca e chuvosa em Vicia sativa L., Avena sativa L., Trifolium

Correspondencia: correodania@gmail.com

Cómo citar este artículo: DANIA FONSECA-LÓPEZ, ISRAEL ALBERTO BOHÓRQUEZMASMELA, CARLOS EDUARDO RODRÍGUEZ-MOLANO, NELSON JOSÉ VIVASQUILA. Efecto del periodo de recuperación en la producción y calidad nutricional de algunas especies forrajeras. Revista Biotecnología en el Sector Agropecuario y Agroindustrial,18(2),2020.135-144, DOI:http://dx.doi.org/10.18684/ BSAA(18)135-144

\section{PALABRAS CLAVE:}

Gramíneas, Leguminosas, Forraje verde, Nutrientes, Suelos.

\section{KEYWORDS:}

Grasses, Legumes, Fresh biomass, Nutrients, Soils.

\section{PALAVRAS-CHAVE:}

Gramínea, Leguminosa, Forragem verde, Nutrient, Solos. 
repens L., Trifolium pratense L., Medicago sativa L., y Dactylis glomerata L. em Boyacá (2700 msnm) em um desenho completamente aleatório, com um arranjo fatorial 4 x 6 que correspondeu a quatro períodos de recuperação (25, 30, 35 e 40 dias) e seis forragens. As variáveis avaliadas foram produção de matéria seca (MS), altura, qualidade nutricional, e disponibilidade de $\mathrm{N}, \mathrm{Ca}$ e $\mathrm{P}$ ao iniciar e ao finalizar o ensaio. Os dados foram analisados com Anova e o teste de Tukey $(p<0,05)$. Observou-se que a produção de forragem (DM) diferiu em todas as espécies entre os períodos de recuperação e o teor de proteína apenas na aveia, as demais variáveis não sofreram alterações estatisticamente. Concluise que o tempo ideal de corte é de 40 dias para trevo vermelho, vicia e aveia, 35 dias para trevo branco e 30 dias para cocksfoot e alfafa.

\section{INTRODUCCIÓN}

El área ocupada por forrajes en Colombia se ha incrementado desde 1960 por influencia de los pastos introducidos como pasto amargo (Brachiaria decumbens), pará (Brachiaria mutica) y guinea (Megathyrsus maximus) y, en los últimos 50 años, el área destinada a su establecimiento se ha duplicado [1] como consecuencia del mejoramiento genético y liberación de semillas evaluadas en campo por diferentes instituciones de carácter público y privado. En Boyacá, ocupan cerca del $60 \%$ del territorio en donde se desarrolla actividad ganadera de lechería, doble propósito y ceba, pero en el ámbito nacional, el departamento es reconocido por su producción lechera debido a su topografía y a su entorno agroecológico [2]. Esta actividad se concentra en municipios ubicados sobre los 2.000 msnm [3] como en la provincia de Sugamuxi (Aquitania, Cuitiva, Firavotba, Iza, Mongui, Tota, Pesca entre otros), provincia Norte (Boavita, Covarachía, San Mateo, Susacon, Tipacoque entre otros), provincia Gutierrez (Chiscas, el Cocuy, Guican, Panqueba entre otros) y provincia de Tundama (Paipa, Floresta, Belen, Busbanzá, Cerinza, Corrales, Santa Rosa de Viterbo entre otros), en donde el forraje con mayor cobertura es kikuyo (Penisetum clandestinum) por su rápida propagación, adapatación a la zona y por sus cualidades nutricionales (21,13\% MS, $14,47 \%$ PC) [4]. Además, otros forrajes de clima frío que suelen encontrarse en los sistemas ganaderos son falsa poa (Holcus lanatus), ryegrass (Lolium spp), trébol rojo (Trifolium pratense), trébol blanco (Trifolium repens), avena forrajera (Avena sativa L.), azul orchoro (Dactylis glomerata), vicia (Vicia sativa), alfalfa (Medicago sativa) y sauco (Sambucus nigra L.) [5]. Estas especies son limitadas por diferentes caracteristicas como humedad, nutrientes, lumiosidad y características del suelo, de modo que estos factores corresponden a condiciones de estrés capaces de ejercer influencia en la respuesta de crecimiento y producción de biomasa en los forrajes, como consecuencia de un efecto directo sobre su metabolismo y fotosíntesis [6] y en la fijación de carbono en materia seca acumulada en las hojas que son el área de captación de la luz [7].

El pastoreo y/o frecuencias de corte tardías o tempranas también repercuten en la calidad nutritiva y producción de forraje, con largos periodos de recuperación se obtiene mayor materia seca de un pasto lignificado que es menos digestible a costa de una reducción considerable en su calidad nutricional y, en el caso contrario, forraje tierno que tiene alto contenido de proteína pero menor producción de biomasa.

El estudio del crecimiento de estas especies en diferentes áreas resulta de importancia económica ya que esto permite adecuar actividades de manejo para lograr una ganadería eco-eficiente [8]. El éxito y la prosperidad de esta actividad está determina por la disponibilidad adecuada y oportuna de alimentos y los forrajes son la fuente más económica para satisfacer las necesidades dietéticas del ganado [9]. Igualmente, el conocimiento del tiempo óptimo para el corte, y las especies con mejor respuesta por zona agroecológica, resultan útiles en condiciones de campo para elegir los mejores cultivares en un sistema productivo en función del lugar y características del suelo. Por ello, el objetivo de este trabajo fue establecer el tiempo óptimo de corte a través de la producción de forraje y valor nutricional de las especies vicia (Vicia sativa L.), avena forrajera (Avena sativa L.), trébol blanco (Trifolium repens L.), trébol rojo (Trifolium pratense L.), alfalfa (Medicago sativa L.) y azul orchoro (Dactylis glomerata L.) y la disponibilidad de nutrientes en el suelo como contribución al conocimiento de la respuesta de crecimiento de forrajes de clima frío.

\section{MÉTODO}

El estudio se realizó en la granja experimental La María de la Universidad Pedagógica y Tecnológica de Colombia ubicada a 2700 m.s.n.m. en el departamento de Boyacá en las coordenadas $5^{\circ} 33^{\prime} 7^{\prime} \mathrm{N}$ y $73^{\circ} 21^{\prime} 23^{\prime \prime} \mathrm{W}$ con una temperatura promedio de 
$13^{\circ} \mathrm{C}$. Las especies forrajeras fueron establecidas en parcelas de $2 \times 3 \mathrm{~m}^{2}$ con espacio entre filas de $50 \mathrm{~cm}$, una vez encalado y fertilizado el suelo de acuerdo a los requerimientos de los forrajes y disponibilidad de nutrientes. Este se clasificó como franco arcilloso, y presentó las siguientes características: $\mathrm{pH}$ 5,74, 2,55 $\mathrm{MO} \%, 16,2 \mathrm{CIC}$ y $1,2 \mathrm{CE} d S \mathrm{~m}^{-1}(5,67 \mathrm{Ca}, 2,64 \mathrm{Mg}$, $1,12 \mathrm{~K}, 0,18 \mathrm{Na}$ en Meq $100 \mathrm{~g}^{-1}$ de suelo) de acuerdo al análisis reportado por el laboratorio de suelos de la misma universidad, a partir de una muestra representativa $(1 \mathrm{Kg}$ ) derivada de la mezcla de 24 sub muestras ha-1 de suelo tomadas al azar en zigzag.

Las parcelas fueron regadas dos veces por semana durante tres horas y luego de 3 meses de establecimiento se realizó un corte de homogeneidad para iniciar el rebrote (día 0). Los tratamientos evaluados fueron cuatro periodos de recuperación: 25, 30, 35 y 40 días, en las especies vicia (Vicia sativa L.), avena forrajera (Avena sativa L.), trébol blanco (Trifolium repens L.), trébol rojo (Trifolium pratense L.), alfalfa (Medicago sativa L.) y azul orchoro (Dactylis glomerata L.) y se analizó su efecto sobre las variables de respuesta producción de forraje verde, materia seca total, altura, valor nutritivo y disponibilidad de nutrientes en el suelo. Para determinar la producción de forraje en materia seca, que es considerado un parámetro que muestra la adaptabilidad de las especies al medio en el que se están evaluando [10], se realizó un corte de $1 \mathrm{~m}^{2}$ de pasto en cada parcela con un marco para aforo y luego se hizo un corte de uniformidad según la metodología propuesta por el CIAT [10] de la red internacional de pastos tropicales (RIEPT). El peso de la materia seca (MS) se calculó con la siguiente expresión [10]:

$\mathrm{MS} / \mathrm{m} 2=\frac{\mathrm{PF} \times \mathrm{ps}}{\mathrm{pf}}$

PF: peso fresco de la muestra

pf: peso fresco de la submuestra

ps= peso seco de la submuestra.

Las muestras se secaron $\left(60^{\circ} \mathrm{C}\right)$ en una estufa marca Memmert ${ }^{\circledR}$ (Karl Kolb, Schwabach, Alemania) y la humedad $(\mathrm{H})$ se calculó por diferencia [11].

La altura se evaluó de 3 plantas seleccionadas al azar en cada parcela y se midió en centímetros desde el suelo hasta el punto más alto de la planta sin estirarla [10].
Para el análisis nutricional se envió una muestra de $500 \mathrm{~g}$ de forraje en sobres de papel puestos dentro de bolsas plásticas individuales al laboratorio AGRILAB compañía limitada, allí se halló en base seca, el contenido de cenizas por incineración a $700^{\circ} \mathrm{C}$, extracto etéreo con éter, fibra cruda, proteína total por Kjeldahl, fósforo según la norma NTC 234 y calcio con ácido nítrico de acuerdo a los procedimientos reportados por el laboratorio. Para establecer el tiempo óptimo de corte se hizo la relación entre la producción de biomasa en materia seca y el contenido proteico.

Cada tratamiento tuvo 3 réplicas y cada parcela correspondió a una unidad experimental para un total de 72 parcelas distribuidas en un diseño completamente al azar con un arreglo factorial $4 \times 6$, donde 4 correspondió a los periodos de recuperación $(25,30$, 35 y 40 días) y 6 al número de especies (Vicia, avena forrajera, trébol rojo, trébol blanco, alfalfa y azul orchoro). Los datos obtenidos en los diferentes periodos de recuperación fueron analizados con un Anova y donde hubo diferencias significativas se aplicó la prueba de Tukey $(p<0,05)$ en el paquete estadístico SPSS statistics versión 19.

\section{RESULTADOS}

Durante el tiempo del ensayo (enero - abril 2019) se observó variación en la temperatura $\left(8,1-22,1^{\circ} \mathrm{C}\right)$ y en la pluviosidad (0,2-4,6 mm/d) (Figura 1) en el área de estudio (3.000 msnm). En marzo y abril se presentaron las lluvias más intensas en promedio 3,3 y 4,6 $\mathrm{mm} / \mathrm{d}$ respectivamente de acuerdo con el reporte de la estación climática de la Universidad Pedagógica y Tecnológica de Colombia.

\section{Forraje verde y biomasa en materia seca}

No se presentaron diferencias estadísticas $(p=0,05)$ en la producción de forraje durante la época de lluvia y seca, lo que se atribuye al uso de riego. Sin embargo, existieron diferencias entre los periodos de recuperación y la producción de forraje verde por especie.

En vicia la mayor producción de forraje verde se observó a los $35\left(16,2 \pm 4,6\right.$ ton ha-1 ${ }^{-1}$ y 40 días $(16,7 \pm 1,4$ ton $\left.\mathrm{ha}^{-1}\right)$ al igual que en alfalfa ( $14 \pm 1,2$ ton $\mathrm{ha}^{-1} ; 14,2 \pm 1,2$ ton $\mathrm{ha}^{-1}$ ). En avena forrajera, trébol rojo y azul orchoro, la mayor producción se obtuvo a los 40 días $(25,7 \pm 3,3$ ton ha-1 ${ }^{-1}, 3,3 \pm 6,3$ ton ha ${ }^{-1}, 3,62 \pm 0,9$ ton ha ${ }^{-1}$ respec- 
tivamente), mientras que en trébol blanco no se presentaron diferencias a los 40, 35 y 30 días (Figura 2). La producción de materia seca presentó diferencias estadísticas entre los periodos de recuperación $(p=0,05)$. No obstante, a los 35 y 40 días el forraje (MS) fue mayor en vicia y trébol blanco, mientras que en avena y trébol rojo a los 40 días; y en alfalfa y azul orchoro a los 30,35 y 40 días sin existir diferencias estadísticas entre estos periodos de recuperación $(p=0,05)$ (Cuadro 1).

En alfalfa, la producción de materia seca es aproximadamente de 12,2 ton ha-1 durante el año [12], en avena de 11,50 a 20,78 ton ha $^{-1}$ en un periodo de corte de 60 a 70 días [9], en azul orchoro de 4,44 a $108 \mathrm{~g} /$ planta con variabilidad por genotipo [13] y entre 0,13 ton ha-1 y 8,04 $\pm 0,19$ ton ha-1 cuando está en mezcla con trébol rojo [14], de modo que los resultados obtenidos alcanzan los rangos reportados, alfalfa con proyección anual de 20,25 ton ha-1, avena 20,1 ton ha-1 ${ }^{-1}$ azul orchoro 7,92 ton ha-1 y vicia 29,2 ton ha-1.

En trébol rojo, los rendimientos de producción de materia seca en el año van desde 3,4 ton ha-1 a 13,9 ton ha-1 lo que se relaciona con la persistencia de la planta a las condiciones ambientales, manejo, pastoreo y al hábito de crecimiento que, a diferencia del trébol blanco que es estolonífero, crece a partir de meristemas apicales de modo que no hay regeneración de las plantas muertas $[15,16]$, por ello esos resultados son menores a los observados en este trabajo con corte cada 40 días que alcanzan los 2,3 ton ha-1 (MS) de modo que se proyectan rendimientos de 20,7 ton $\left.^{-1} a^{-1} \mathrm{MS}\right)$ al año.

Figura 1. Temperatura máxima, temperatura mínima y pluviosidad durante el periodo comprendido entre enero y abril.

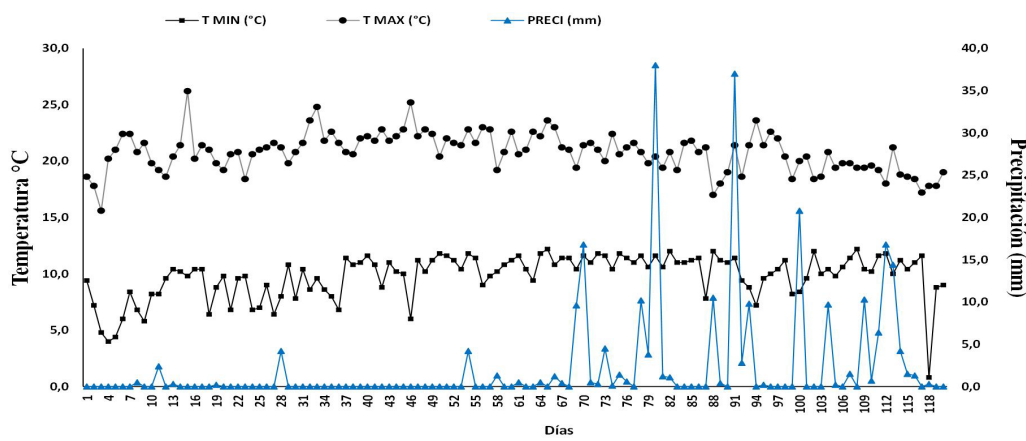

Figura 2. Producción de forraje verde durante los diferentes periodos de recuperación en las especies vicia (Vi), avena forrajera (AV), trébol rojo (TG), trébol blanco (TRG), alfalfa (AL) y azul orchoro (AZ).

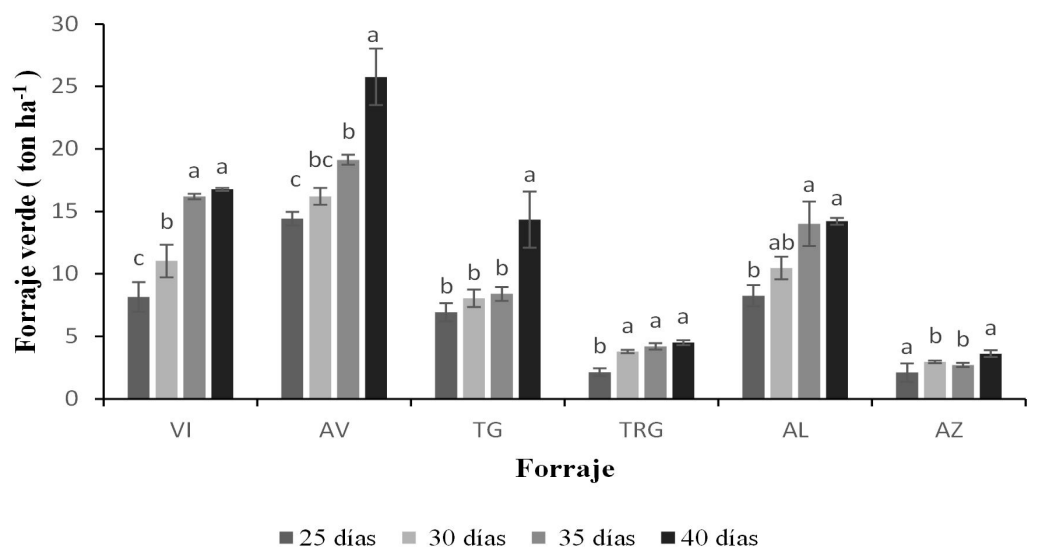

Letras distintas indican diferencias significativas por la prueba de Tukey $(p=0,05)$. Las barras representan el error estándar.

Cuadro 1. Producción de forraje ton ha-1 materia seca (MS)

\begin{tabular}{|l|c|c|c|c|}
\hline \multirow{2}{*}{\multicolumn{1}{c|}{ Especie }} & \multicolumn{4}{|c|}{ Periodo de recuperación(Días) } \\
\cline { 2 - 5 } & 25 & 30 & 35 & 40 \\
\hline Vicia sativa L. & $1,37 \pm 0,1 \mathrm{c}$ & $1,9 \pm 0,1 \mathrm{~b}$ & $3,5 \pm 0,1 \mathrm{a}$ & $3,25 \pm 0,1 \mathrm{a}$ \\
\hline Avena sativa L. & $2,61 \pm 0,2 \mathrm{c}$ & $3,2 \pm 0,1 \mathrm{c}$ & $4,4 \pm 0,1 \mathrm{~b}$ & $6,7 \pm 0,1 \mathrm{a}$ \\
\hline Trifolium pratense L. & $1,37 \pm 0,1 \mathrm{~b}$ & $1,5 \pm 0,1 \mathrm{~b}$ & $1,5 \pm 0,2 \mathrm{~b}$ & $2,3 \pm 0,1 \mathrm{a}$ \\
\hline Trifolium repens L. & $0,41 \pm 0,2 \mathrm{~b}$ & $0,67 \pm 0,1 \mathrm{~b}$ & $0,77 \pm 0,1 \mathrm{a}$ & $0,72 \pm 0,2 \mathrm{ab}$ \\
\hline Medicago sativa L. & $1,17 \pm 0,1 \mathrm{~b}$ & $2,38 \pm 0,1 \mathrm{a}$ & $2,2 \pm 0,3 \mathrm{a}$ & $2,25 \pm 0,2 \mathrm{a}$ \\
\hline Dactylis glomerata L. & $0,4 \pm 0,1 \mathrm{~b}$ & $0,84 \pm 0,1 \mathrm{a}$ & $0,7 \pm 0,1 \mathrm{a}$ & $0,88 \pm 0,1 \mathrm{a}$ \\
\hline
\end{tabular}

Letras distintas por especie indican diferencias significativas con la prueba de Tukey $(P=0,05)$

Mientras que en trébol blanco con 9 rotaciones al año es decir pastoreos cada 40 días se ha logrado producciones de 13,03 a 14,1 ton $\mathrm{ha}^{-1}$ [17] valores supe- riores a lo obtenido que en el año se aproxima a 6,48 ton $\mathrm{ha}^{-1}$ de modo que esta especie no tuvo buen rendimiento bajo las condiciones evaluadas, además, el con- 
tenido de materia seca fue menor al reportado de $266 \mathrm{~g} \mathrm{MS} \mathrm{kg}^{1}$ de forraje verde [17].

\begin{abstract}
Altura
El periodo de recuperación influyó significativamente en la altura alcanzada entre los 25 y 40 días en vicia, avena, alfalfa y pasto azul $(p=0,05)$ pero no en trébol rojo y blanco. En vicia la altura estuvo entre $81 \pm 6,24$ y $116 \pm 7,5$ $\mathrm{cm}$, en avena forrajera de $84,66 \pm$ 9,5 a $129 \pm 1,5 \mathrm{~cm}$, en trébol rojo de $72,3 \pm 7,5$ a $80,6 \pm 6,02 \mathrm{~cm}$, en trébol blanco de $26,7 \pm 1,5$ a $28,3 \pm 1,5 \mathrm{~cm}$, en alfalfa de 70,6 $\pm 3,05$ a $85,3 \pm 0,5 \mathrm{~cm}$ y en azul orchoro de 25,13 $\pm 1,6$ a 29,6 \pm $1,5 \mathrm{~cm}$ (Figura 3).
\end{abstract}

Estos resultados coinciden con las alturas reportadas en literatura entre 59,83 y $83,33 \mathrm{~cm}$ en alfalfa, 99,9 y $123,8 \mathrm{~cm}$ en avena, $56,9 \mathrm{~cm}$ en azul orchoro, en trébol rojo hasta $97 \mathrm{~cm}$, en trébol blanco entre 10 y $30 \mathrm{~cm}$ y en vicia hasta $120 \mathrm{~cm}[9,13,18]$.

\section{Características nutricionales del forraje y químicas del suelo}

Se presentaron diferencias estadísticas $(p=0,05)$ en el porcentaje de materia seca entre los periodos de recuperación en todas las especies evaluadas excepto en los tréboles. Para el contenido de proteína solo avena presentó diferencia significativa entre los cortes. El resto de las características nutricionales evaluadas (contenido de cenizas, extracto etéreo, fibra bruta, calcio y fósforo) no cambiaron estadísticamente (Cuadro 2). A los 25 días se presentaron las proteínas más altas excepto para el trébol blanco que fue a los 30 y 35 días, pero

Figura 3. Altura durante diferentes periodos de recuperación en las especies vicia (VI), avena forrajera (AV), trébol rojo (TG), trébol blanco (TRG), alfalfa (AL) y azul orchoro (AZ).

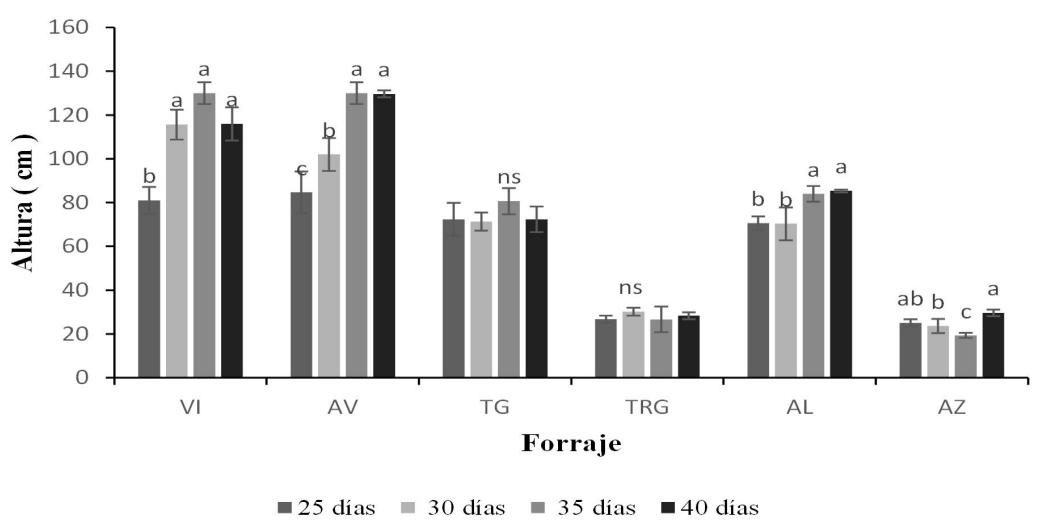

Letras distintas indican diferencias significativas por la prueba de Tukey $(P=0,05)$. Las barras representan el error estándar.

al relacionar el forraje producido con las características nutricionales se observó que en vicia a los 35 días se obtuvo 0,93 ton ha-1 de proteína, es decir 0,12 ton ha $^{-1}$ adicionales que a los 40 días, sin embargo, esta especie por lo general se siembra con avena forrajera que a su vez expresa su mayor potencial a los 40 días de rebrote con 1,23 ton ha-1 de proteína, por ello, se debe considerar la siembra por separado de estas especies, y en caso de asociación el corte optimo es cada 40 días.

Esta misma frecuencia se recomienda para trébol rojo que alcanza 0,59 ton ha-1 de proteína, lo que a su vez representa 0,21 ton ha ${ }^{-1}$ adicional respecto a los 35 días, y también para alfalfa con 0,58 ton ha-1, aunque es posible realizar cortes a los 30 y 35 días ya que la diferencia no es significativa $\left(0,016\right.$ ton $\left.\mathrm{ha}^{-1}\right)$.

En trébol blanco y azul ochoro la mayor producción de proteína en relación con el forraje se obtuvo a los 30 y 35 días respectivamente con un valor aproximado de 1,18 ton $\mathrm{ha}^{-1}$ para ambas especies. El rendimiento de este parámetro nutricional por unidad de área se relaciona con la obtención de materia seca, de modo que en trébol rojo se ha encontrado proteínas que oscilan entre 1,6 a 2,9 ton $\mathrm{ha}^{-1}$ el primer año y desde 1,1 a 1,9 ton ha-1 al cuarto año [15], valores superiores a lo observado en este estudio a diferencia de trébol blanco cuyo contenido proteico fue mayor a $232,4 \mathrm{~g} \mathrm{~kg}^{-1}$ (Ms) con 40 días de corte [17]. Mientras que en alfalfa se estiman de 0,25 a 0,35 ton ha-1 de nitrógeno [12] que se encuentra concentrado en un $70 \%$ en las hojas [19] en general puede variar entre 22,13 y $27,7 \%$ de PC $[12,20]$. Por otro lado, en avena forrajera es común encontrar de 7,08 a 10,66 \% PB [9], en vicia sativa 347 a $374 \mathrm{~g} \mathrm{~kg}^{-1}$ de MS [21] y cuando se realizan mezclas de pasto azul orchoro y trébol blanco entre 17,5 y $22,2 \%$ PB [14] calidad nutricional aceptable.

Por otro lado, el pasto contiene minerales esenciales para el ganado, pero sus concentraciones están influenciadas por factores climáticos, del suelo y agronómicos que conducen a situaciones difíciles para la suplementación 
Cuadro 2. Características nutricionales durante diferentes periodos de recuperación en las especies vicia (VI), avena forrajera (AV), trébol rojo (TG), trébol blanco (TRG), alfalfa (AL) y azul orchoro (AZ).

\begin{tabular}{|c|c|c|c|c|c|c|c|}
\hline Característica & Día & VI & AV & TG & TRG & $\mathrm{AL}$ & $A Z$ \\
\hline \multirow{4}{*}{$H(\%)$} & 25 & $83,1 \pm 0,2 a$ & $81,8 \pm 0,3 a$ & $80,2 \pm 2,7$ & $80,8 \pm 1,3$ & $85,7 \pm 1,1 \mathrm{a}$ & $80,6 \pm 1,4 a$ \\
\hline & 30 & $82,7 \pm 0,1 \mathrm{a}$ & $80,3 \pm 0,2 a$ & $81,6 \pm 0,9$ & $82,1 \pm 0,4$ & $77,2 \pm 0,3 b$ & $71,3 \pm 0,8 b$ \\
\hline & 35 & $77,9 \pm 0,8 b$ & $76,8 \pm 0,8 b$ & $81,7 \pm 1,3$ & $81,7 \pm 2,4$ & $85,2 \pm 0,6 a$ & $73,8 \pm 2,5 b$ \\
\hline & 40 & $80,5 \pm 0,5 c$ & $72,9 \pm 0,3 c$ & $83,6 \pm 0,8$ & $83,9 \pm 0,7$ & $83,1 \pm 1,1 a$ & $75,5 \pm 1,2 b$ \\
\hline \multirow{4}{*}{ MS (\%) } & 25 & $16,8 \pm 0,3 c$ & $18,1 \pm 0,3 c$ & $19,8 \pm 2,7$ & $19,2 \pm 1,3$ & $14,3 \pm 1,1 b$ & $19,4 \pm 1,4 \mathrm{c}$ \\
\hline & 30 & $17,2 \pm 0,1 b$ & $19,7 \pm 0,2 c$ & $18,4 \pm 0,9$ & $17,9 \pm 0,4$ & $15,7 \pm 0,6 b$ & $28,7 \pm 0,8 a$ \\
\hline & 35 & $19,4 \pm 0,5 b$ & $23,2 \pm 0,8 b$ & $18,3 \pm 1,3$ & $18,3 \pm 2,4$ & $15,8 \pm 1,5 b$ & $26,1 \pm 2,5 b$ \\
\hline & 40 & $22,1 \pm 0,8 a$ & $27 \pm 0,3 a$ & $16,3 \pm 0,9$ & $16,1 \pm 0,7$ & $22,7 \pm 0,4 a$ & $24,5 \pm 0,9 b$ \\
\hline \multirow{4}{*}{ CE (\%) } & 25 & $8,6 \pm 0,1$ & $8,6 \pm 0,1$ & $9,8 \pm 0,1$ & $12 \pm 1,5$ & $9,1 \pm 0,8$ & $9,5 \pm 0,8$ \\
\hline & 30 & $7,6 \pm 0,1$ & $8,5 \pm 0,1$ & $7,4 \pm 0,6$ & $11,1 \pm 0,1$ & $9,1 \pm 1,1$ & $10,2 \pm 0,7$ \\
\hline & 35 & $8,1 \pm 0,1$ & $8,6 \pm 0,1$ & $9,7 \pm 0,7$ & $10,9 \pm 0,3$ & $11,8 \pm 0,1$ & $10,9 \pm 1,1$ \\
\hline & 40 & $9,2 \pm 0,1$ & $10,7 \pm 0,2$ & $10 \pm 0,2$ & $9,7 \pm 0,1$ & $9,7 \pm 0,2$ & $8,7 \pm 0,4$ \\
\hline \multirow{4}{*}{ EE (\%) } & 25 & $2,6 \pm 0,1$ & $2,1 \pm 0,1$ & $2,5 \pm 0,2$ & $2,3 \pm 0,2$ & $1,7 \pm 0,1$ & $2,5 \pm 0,8$ \\
\hline & 30 & $2,1 \pm 0,1$ & $2,4 \pm 0,1$ & $1,8 \pm 0,1$ & $2,6 \pm 0,1$ & $2,2 \pm 0,3$ & $2,2 \pm 0,4$ \\
\hline & 35 & $2,6 \pm 0,1$ & $2,6 \pm 0,1$ & $1,8 \pm 0,2$ & $1,8 \pm 0,1$ & $1,7 \pm 0,1$ & $2,6 \pm 0,1$ \\
\hline & 40 & $2,3 \pm 0,1$ & $2,1 \pm 0,2$ & $2,5 \pm 0,1$ & $2,2 \pm 0,1$ & $2,3 \pm 0,1$ & $2,9 \pm 0,6$ \\
\hline \multirow{4}{*}{ FB (\%) } & 25 & $25,2 \pm 0,9$ & $19,4 \pm 2,2$ & $14,5 \pm 0,5$ & $13,6 \pm 1,2$ & $20,6 \pm 1,6$ & $26,1 \pm 0,4$ \\
\hline & 30 & $27,5 \pm 4,6$ & $20,1 \pm 1,2$ & $19 \pm 3,1$ & $16,9 \pm 4,3$ & $22,1 \pm 1,2$ & $26,2 \pm 0,2$ \\
\hline & 35 & $28,7 \pm 2,1$ & $24,3 \pm 0,9$ & $14,1 \pm 1,7$ & $18,5 \pm 0,5$ & $21,9 \pm 1,1$ & $25,4 \pm 0,5$ \\
\hline & 40 & $29,7 \pm 1,7$ & $25,2 \pm 1,5$ & $16,3 \pm 0,5$ & $18,1 \pm 1,9$ & $22,2 \pm 0,3$ & $25,1 \pm 1,5$ \\
\hline \multirow{4}{*}{ PB (\%) } & 25 & $26 \pm 0,41$ & $17,8 \pm 0,9 a$ & $28,8 \pm 2,9$ & $28,9 \pm 1,1$ & $26,4 \pm 2,5$ & $22,8 \pm 2,7$ \\
\hline & 30 & $24,8 \pm 0,3$ & $13,2 \pm 0,4 b$ & $27,6 \pm 3,3$ & $30,6 \pm 1,1$ & $25,4 \pm 1,9$ & $22,3 \pm 2,1$ \\
\hline & 35 & $23 \pm 1,3$ & $12,3 \pm 0,5 b$ & $24,6 \pm 1,5$ & $30,6 \pm 2,3$ & $25,7 \pm 0,5$ & $20,5 \pm 1,5$ \\
\hline & 40 & $22,5 \pm 1,5$ & $11,8 \pm 0,4 b$ & $24,2 \pm 0,9$ & $26,9 \pm 0,2$ & $23,4 \pm 1,5$ & $19,1 \pm 1,2$ \\
\hline \multirow{4}{*}{$\mathrm{P}(\%)$} & 25 & $0,27 \pm 0,1$ & $0,22 \pm 0,1$ & $0,35 \pm 0,1$ & $0,35 \pm 0,1$ & $0,41 \pm 0,1$ & $0,22 \pm 0,1$ \\
\hline & 30 & $0,34 \pm 0,1$ & $0,23 \pm 0,1$ & $0,27 \pm 0,1$ & $0,28 \pm 0,1$ & $0,3 \pm 0,1$ & $0,2 \pm 0,1$ \\
\hline & 35 & $0,3 \pm 0,1$ & $0,39 \pm 0,1$ & $0,31 \pm 0,1$ & $0,3 \pm 0,1$ & $0,4 \pm 0,1$ & $0,26 \pm 0,1$ \\
\hline & 40 & $0,5 \pm 0,5$ & $0,92 \pm 0,5$ & $0,4 \pm 0,1$ & $0,4 \pm 0,1$ & $0,3 \pm 0,1$ & $0,69 \pm 0,2$ \\
\hline \multirow{4}{*}{$\mathrm{Ca}(\%)$} & 25 & $0,87 \pm 0,1$ & $0,35 \pm 0,1$ & $1,6 \pm 0,1$ & $1,1 \pm 0,1$ & $1,6 \pm 0,1$ & $0,6 \pm 0.1$ \\
\hline & 30 & $0,9 \pm 0,1$ & $0,4 \pm 0,1$ & $1,6 \pm 0,1$ & $1,2 \pm 0,1$ & $2,2 \pm 0,5$ & $0,5 \pm 0,1$ \\
\hline & 35 & $0,98 \pm 0,1$ & $0,52 \pm 0,2$ & $1,5 \pm 0,1$ & $1,1 \pm 0,1$ & $2,2 \pm 0,4$ & $0,5 \pm 0,1$ \\
\hline & 40 & $0,93 \pm 0,1$ & $0,52 \pm 0,1$ & $1,8 \pm 0,1$ & $1,2 \pm 0,1$ & $2,3 \pm 0,4$ & $0,6 \pm 0,1$ \\
\hline
\end{tabular}

$\mathrm{H}$ : humedad, MS: materia seca, CE: cenizas, EE: extracto etéreo, FB: fibra bruta, PB: proteína bruta, P: fósforo, Ca: calcio. Letras diferentes indican diferencias significativas con la prueba de Tukey $(P=0,05)$.

de minerales de los animales [22], en avena forrajera es común encontrar entre 9,08 y $14,34 \%$ de contenido mineral [9] y en alfalfa valores de 0,35 a 0,41 de fosforo (\% P) y de 2,43 a 1,96 en calcio (\%Ca) [19].

No hubo diferencias significativas entre el contenido de calcio y fosforo en los forrajes, pero, las diferencias numéricas muestran un comportamiento general de disminución respecto al tiempo (Cuadro 3), esto mismo se reporta en trébol rojo en donde el contenido de calcio en el suelo pasó de 1.880 (10 $\mathrm{cm})$ a $1.210 \mathrm{mg} \mathrm{kg}^{-1}$ de $\mathrm{MS}(40 \mathrm{~cm})$ y en fósforo de 37 $(10 \mathrm{~cm})$ a $10 \mathrm{mg} \mathrm{kg}^{-1} \mathrm{de} \mathrm{MS}(40 \mathrm{~cm})$ [23].

Lo que se debe a la absorción de estos nutrientes por la planta, el calcio para la formación de paredes celulares, como cofactor de algunas enzimas que están involucradas en la hidrólisis de ATP y como segundo mensajero en la regulación metabólica que media los procesos de estrés y desarrollo [6,24,25,26]. Mientras que el fósforo es utilizado por la planta como cofactor durante la fijación de nitrógeno por ello el 
Cuadro 3. Características químicas del suelo al comienzo y final del periodo de recuperación.

\begin{tabular}{|c|c|c|c|c|c|c|c|}
\hline Característica & Días & VI & AV & TG & TRG & $\mathrm{AL}$ & $A Z$ \\
\hline \multirow{2}{*}{$\mathrm{pH}$} & 0 & 6,07 & 5,48 & 5,95 & 6 & 6,14 & 5,52 \\
\hline & 40 & 6,05 & 5,75 & 5,94 & 6,06 & 6,15 & 5,6 \\
\hline \multirow{2}{*}{ MO (\%) } & 0 & 3,36 & 2,91 & 3,47 & 3,31 & 3,65 & 3,54 \\
\hline & 40 & 2,52 & 2,53 & 3,38 & 2,79 & 3,34 & 3,19 \\
\hline \multirow{2}{*}{$\mathrm{N}(\%)$} & 0 & 0,162 & 0,141 & 0,168 & 0,16 & 0,177 & 0,183 \\
\hline & 40 & 0,122 & 0,122 & 0,163 & 0,135 & 0,162 & 0,102 \\
\hline \multirow{2}{*}{$\mathrm{Ca}\left(\mathrm{mg} \mathrm{kg}^{-1} \mathrm{MS}\right)$} & 0 & 1500 & 1340 & 1390 & 1460 & 1380 & 1350 \\
\hline & 40 & 1260 & 510 & 1370 & 1500 & 1320 & 1285 \\
\hline \multirow{2}{*}{$\mathrm{P}\left(\mathrm{mg} \mathrm{kg}^{-1} \mathrm{MS}\right)$} & 0 & 105 & 113 & 107 & 89,1 & 105 & 98,5 \\
\hline & 40 & 79,3 & 76,5 & 101 & 72,6 & 94,7 & 72,8 \\
\hline
\end{tabular}

MO: materia orgánica, N: nitrógeno, Ca: calcio, P: fósforo. Vicia (VI), avena forrajera (AV), trébol rojo (TG), trébol blanco (TRG), alfalfa $(A L)$ y azul orchoro (AZ).

requerimiento de fósforo en las leguminosas es más alto, además, influye en el desarrollo de los nódulos, forma la estructura de los ácidos nucleicos, nucleótidos, fosfolípidos y ATP [18]. Aunque no hubo variaciones relevantes en el $\mathrm{pH}$ del suelo, las especies evaluadas se adaptaron bien lo que se debe a la resistencia de estos forrajes a condiciones de acidez ligera, excepto la alfalfa, por ello es común que azul orchoro se desarrolle en suelos con $\mathrm{pH}$ de 6,8 y contenido de materia orgánica de 1,2\% [13,22] al igual que trébol blanco y trébol rojo aunque también pueden desarrollarse a pH de 6,4 y 6,1 respectivamente o en rangos inferiores a pH 5 en suelos compactos pobremente drenados $[15,17,27]$, por ello son especies potenciales en los sistemas ganaderos ya que se adaptan a diferentes condiciones y toleran la presencia de metales pesados como el $\mathrm{Pb}$, además contribuyen a la fertilidad del suelo por la fijación de $\mathrm{N}_{2}$ en simbiosis con diazótrofos [28].

Como se mencionaba, alfalfa es el único forraje evaluado con condicionamiento de $\mathrm{pH}$ en el suelo ya que su desarrollo óptimo se encuentra entre 6,4 y 6,85 [18] y por debajo de 4,8 su crecimiento es difícil y se reduce significativamente [19] aunque puede tolerar rangos de 7,5 a 7,74 [18] de modo que el pH de 6,14 del suelo donde se realizó el trabajo fue aceptable. Este parámetro es importante ya que la neutralidad facilita la disponibilidad de elementos como el Py el K [29] de modo que con rangos de $\mathrm{pH}$ similares se ha detectado niveles de $\mathrm{P}$ de $61 \mathrm{mg} \mathrm{kg}^{-1}$ [19].

\section{CONCLUSIONES}

El periodo de recuperación en Vicia sativa L., Avena sativa L., Trifolium repens L., Trifolium pratense L., Medicago sativa L., y Dactylis glomerata L. influye en la producción de biomasa (verde y seca) y en su calidad nutricional con diferencias significativas en el contenido de proteína, que es alto a los 25 días de rebrote y disminuye gradualmente.

En trébol rojo, avena forrajera y vicia se logró una producción óptima de materia seca de 2,3 ton ha- ${ }^{-1}$, 3,25 ton ha-1 y 6,7 ton ha-1 a los 40 días respectivamente con un contenido proteico de $24,2 \%, 11,8 \%$ y $22,5 \%$ por especie.

En alfalfa la mayor producción de forraje se produjo a los 30,35 y 40 días sin diferencia significativa entre ellos, no obstante se puede sugerir que el momento óptimo de corte es a los 35 días ya que se relaciona con el mayor porcentaje de PB (25,7\%).

Trébol blanco presentó la mayor producción de forraje a los 35 días $(0,77$ ton ha-1) con buen valor de PB $(30,6 \%)$ por lo que se recomienda esta fecha de corte para pastoreo.

Azul orchoro presentó la mayor producción de forraje a los 30, 35 y 40 días sin existir diferencias estadísticas $\left(0,7\right.$ ton $\left.\mathrm{ha}^{-1}\right)$ pero se recomienda realizar pastoreo a los 30 días ya que en este corte se observó la mayor PB (22,3\%) 
El contenido de calcio y fósforo en las especies no tuvo variación significativa entre periodos de recuperación, sin embargo, en el suelo su contenido se redujo gradualmente por efecto de su absorción e incorporación en la estructura de la planta, esto mismo se observó con el nitrógeno disponible en el suelo.

\section{AGRADECIMIENTOS}

Los autores manifiestan agradecimientos a la Universidad Pedagógica y Tecnológica por la financiación, al semillero del grupo de investigación en Bioquímica y Nutrición Animal (GIBNA) por el apoyo operativo y al grupo de Investigación Nutrición Agropecuaria (NUTRIFACA) de la Universidad del Cauca con el que se realizó alianza.

\section{REFERENCIAS}

[1] AUSDAL, S. Pasture, profit, and power: An environmental history of cattle ranching in Colombia. Geoforum, 40(5), 2009. doi:https://doi. org/10.1016/j.geoforum.2008.09.012

[2] DEAQUIZ-MEJIA, J.E., y ZAMBRANO-VARGAS, S.M. Diagnóstico situacional y ambiental de la cadena láctea del Departamento de Boyacá. In Vestigium Ire, 5(1), 2012, p. 37-46.

[3] CARULLA, J.E. y ORTEGA, E. Sistemas de producción lechera en Colombia: retos y oportunidades. Archivos Latinoamericanos de Producción Animal, 24(2), 2016, p. 83-87

[4] FONSECA-LÓPEZ, D. y BORRAS-SANDOVAL, L.M. Evaluación del efecto de la papa fresca incluida en un alimento para vacas holstein sobre la producción y calidad de la leche. Ciencia y Agricultura, 11(1), 2014, p. 55-65. doi:https:// doi.org/10.19053/01228420.3488

[5] RODRÍGUEZ-MOLANO, C., FONSECA-LÓPEZ, D., NIÑO-MONROY, L.E., SALAMANCA-LÓPEZ, A.E., HOYOS-CONCHA, J.L., y OTERO-RAMÍREZ, I.D. Caracterización nutricional y de producción de biomasa Sambucus peruviana, Sambucus nigra y Morus alba en un banco forrajero. Ciencia en Desarrollo, 10(2), 2019. doi:https://doi.org/10.19053/01217488. v10.n2.2019.9098

[6] HASHEM, A., ABD-ALLAH, E., ALQARAWI, A., MALIK, J., WIRTH, S. and EGAMBERDIEVA, D. Role of calcium in AMF-mediated alleviation of the adverse impacts of cadmium stress in Bas- sia indica. Saudi Journal of Biological Sciences, 26(4), 2019, p. 828 - 838. doi:https://doi.org/10.1016/j.sjbs.2016.11.003

[7] BAR-EVEN, A. Daring metabolic designs for enhanced plant carbon fixation alternative carboxylation replacing Rubisco. Plant Science, 273, 2018, p. 71-83. doi:https://doi.org/10.1016/j.plantsci.2017.12.007

[8] MORALES-VELASCO, S., VIVAS-QUILA, N.J. y TERAN-GOMEZ, V.F. Ganaderia eco-eficiente y la adaptaciónal cambio climatico. Biotecnología en el Sector Agropecuario y Agroindustrial, 14(1), 2016, p. 135-144. doi:http://dx.doi. org/10.18684/BSAA(14)135-145

[9] BILAL, M., AYUB, M., TARIQ, M., TAHIR, M. and NADEEM, M.A. Dry matter yield and forage quality traits of oat (Avena sativa L.) under integrative use of microbial and synthetic source of nitrogen. Journal of Saudi Society of Agricultural Sciences, 16(3), 2017, p. 236-241. doi:https://doi.org/10.1016/j.jssas.2015.08.002

[10] COLOMBIA. CENTRO INTERNACIONAL DE AGRICULTURA TROPICAL (CIAT). Manual para la evaluación agronómica. Red internacional de evaluación de pastos tropicales. Cali (Colombia): 1982, $168 \mathrm{p}$.

[11] FONSECA-LÓPEZ, D., SAAVEDRA-MONTAÑÉZ, G. y RODRÍGUEZ-MOLANO, C.E. Elaboración de un alimento para ganado bovino a base de zanahoria (Daucus carota L.) mediante fermentación en estado sólido como una alternativa ecoeficiente. Revista Colombiana de Ciencias Hortícolas, 12(1), 2018, p. 175-182, 2018. doi:https://doi.org/10.17584/ rcch.2018v12i1.7416

[12] HELALIA, A.M. and AL-TAHIR, O.A. The influence of irrigation water salinity and fertilizer management on the yield of Alfalfa (Medicago sativa L.). Agricultural water Management, 1, 1996, p. 105-114

[13] ZHOURI, L., KALLIDA, R., SHAIMI, N., BARRE, P., FLORENCE, V., GABOUN, F. and FAKIRI, M. Evaluation of cocksfoot (Dactylis glomerata L.) population for drought survival and behavior. Saudi Journal of Biological Sciences, 26(1), 2019, p. 49-56. doi:https://doi.org/10.1016/j.sjbs.2016.12.002

[14] CORSON, M.S., ROTZ, C.A., SKINNER, R.H. and SANDERSON, M.A. Adaptation and evaluation of the integrated farm system model to simulate temperate multiple-species pastures. Agricultural Systems, 94, 2007, p. 502-508. doi:https://doi.org/10.1016/j.agsy.2007.01.003 
[15] MARSHALL, H.A., COLLINS, R.P., VALE, J. and LOWE, M. Improved persistence of red clover (Trifolium pratense L.) increases the protein supplied by red clover/grass swards grown over four harvest years. European Journal Agronomy, 89, 2017, p. 38-45. doi.https://doi.org/10.1016/j.eja.2017.06.006

[16] NEGRÓN, M., LÓPEZ, I. and DÖRNER, J. Consequences of intensive grazing by dairy cows of contrasting live weights on volcanic ash topsoil structure and pasture dynamics. Soil \& Tillage Research, 189, 2018, p. 88-97. doi:https://doi. org/10.1016/j.still.2018.12.025

[17] EGAN, M., GALVIN, N. and HENNESSY, D. Incorporating white clover (Trifolium repens L.) into perennial ryegrass (Lolium perenne L.) swards receiving varying levels of nitrogen fertilizer : Effects on milk and herbage production. Journal of Dairy Science, 101(4), 2018, p. 3412-3427. doi. https:// doi.org/10.1017/S0021859616000952

[18] AL-KAHTANI, S., TAHA, E. and AL-ABDULSALAM, $M$. Alfalfa (Medicago sativa $L$.) seed yield in relation to phosphorus fertilization and honeybee pollination. Saudi Journal Biological Sciences, 24(5), 2017, p. 1051-1055. doi:https://doi.org/10.1016/j. sjbs.2016.12.009

[19] MIELMANN, A., BOTHMA, C., HUGO, A. and HUGO, C.J. A comparative study of the chemical composition of lucerne (Medicago sativa L.) and spinach beet (Beta vulgaris var . cicla L.). South African Journal of Botany, 108(1), 2017, p. 8-14. doi:https://doi.org/10.1016/j.sajb.2016.09.006

[20] CONTRERAS, J.L., CORDERO, A.G., CURASMA, J., THIMOTHÉE, J.A. y DEL SOLAR, J. Influencia ambiental sobre el valor nutritivo de alfalfa (Medicago sativa L.) en los andes Peruanos. Compendio de Ciencias Veterinarias, 9(1), 2019, p. 7-14. doi:https://dx.doi.org/10.18004/ compend.cienc.vet.2019.09.01.07-14

[21] HUANG, Y., LI, R., COULTER, A., ZHANG, Z. and NAN, Z. Comparative grain chemical composition, ruminal degradation in vivo, and intestinal digestibility in vitro of Vicia sativa L. varieties. Animals, 2(2), 2019. doi:https://doi.org/10.3390/ani9050212

[22] SCHLEGEL, P., WYSS, U., ARRIGO, Y. and HESS, H.D. Mineral concentrations of fresh herbage from mixed grassland as influenced by botanical composition, harvest time and growth stage. Animal Feed Science and Technology, 219, 2016, p. 226-233. doi.https://doi. org/10.1016/j.anifeedsci.2016.06.022
[23] NIEMI, R.M., VEPSALAINEN, M., WALLENIUS, K., SIMPANEN, S., ALAKUKKU, L. and PIETOLA, L. Temporal and soil depth-related variation in soil enzyme activities and in root growth of red clover (Trifolium pratense) and timothy (Phleum pratense) in the field. Applied Soil Ecology, 30, 2005, p. 113-125. doi:https://doi.org/10.1016/j.apsoil.2005.02.003

[24] YUAN, P., TANAKA, K., DU, L. and POOVAIAH, B. Calcium signaling in plant autoimmunity : a guard model for AtSR1/CAMTA3-mediated immune response. Molecular Plant, 11(5), 2018, p. 637-639, doi.https://doi.org/10.1016/ j.molp.2018.02.014

[25] EDEL, K.H., MARCHADIER, E., BROWNLEE, C., KUDLA, J. and HETHERINGTON, A.M. The evolution of calcium-based signalling in plants. Current Biology, 27, 2017, p. 6-11. e: doi.http:// dx.doi.org/10.1016/j.cub.2017.05.020

[26] ZHU, M., YU, J., TANG, W., FAN, S., BAI, M. and $\mathrm{CHEN}, \mathrm{M}$. Role of calcium in regulating anthocyanin accumulation in 'Manicure Finger' grape berries. Scientia Horticculturae, 256, 2019, p. 1-7. https:// doi.org/10.1016 / j.scienta.2019.108585

[27] ZARZA, R., REBUFFO, M., LA, A. and BALZARINI, $M$. Plant density in red clover (Trifolium pratense $\mathrm{L}$.) pastures as an early predictor of forage production. European Journal Agronomy, 101, 2018, p. 193199. doi:https://doi.org/10.1016/j.eja.2018.10.004

[28] MCKENNA, P., CANNON, N., CONWAY, J. and DOOLEY, J. The use of red clover (Trifolium pratense) in soil fertility-building : A Review. Field Crops Research, 221, 2018, p. 38-49. doi:https://doi.org/10.1016/j.fcr.2018.02.006

[29] TIBBETT, M., GIL-MARTÍNEZ, M., FRASER, T., GREEN, L. D., DUDDIGAN, S., OLIVEIRA, V. H., RAULUND-RASMUSSEN, K., SIZMUR, T. and DIAZ, A. Long-term acidification of $\mathrm{pH}$ neutral grasslands affects soil biodiversity, fertility and function in a heathland restoration. Catena, 180, 2019, p. 401-415. doi:https://doi.org/10.1016/j. catena.2019.03.013 


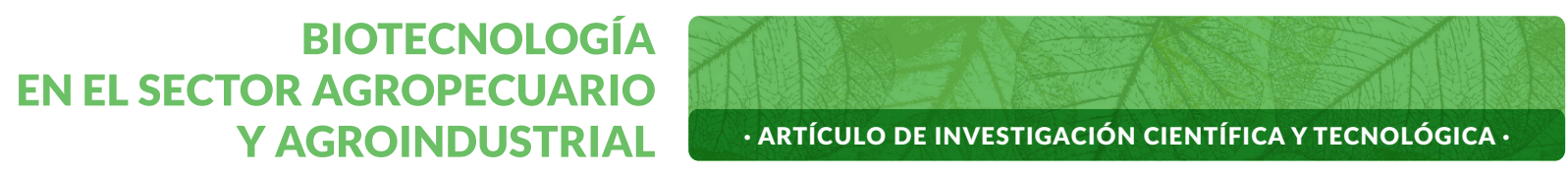

\title{
Efecto microbiológico de recubrimiento modificado por vía ácida sobre el tomate larga vida*
}

\section{Microbiological effect of coating modified by acid via on the long tomato life}

\section{Efeito microbiológico do revestimento de ácido amplificado na vida de longo tomate}

\author{
NELSY HOYOS-YELA ${ }^{1}$, ROCÍO PÉREZ-IMBACHÍ2, SANDRA PATRICIA PAZ-PEÑA ${ }^{3}$, \\ SILVIO ANDRÉS MOSQUERA-SÁNCHEZ ${ }^{4}$
}

Historial del Artículo

Recibido para evaluación: 22 de Mayo 2019.

Aprobado para publicación: 1 de Mayo 2020.

* Proyecto de investigación de origen: "Efecto de la aplicación de un recubrimiento de almidón de yuca modificado por vía ácida sobre el tomate larga vida (Solanum lycopersicum). Financiación: Universidad del Cauca. Culminado el 22 de febrero de 2017.

1 Universidad del Cauca, Facultad de Ciencias Agrarias, Grupo Ciencia y Tecnología de Biomoléculas de Interés Agroindustrial (CYTBIA). Ingeniera Agroindustrial. Popayán, Colombia. https://orcid.org/0000-0002-9854-473X

2 Universidad del Cauca, Facultad de Ciencias Agrarias, Grupo Ciencia y Tecnología de Biomoléculas de Interés Agroindustrial (CYTBIA). Ingeniera Agroindustrial. Popayán, Colombia. https://orcid.org/0000-0001-7913-6004

3 Universidad del Cauca, Facultad de Ciencias Agrarias, Grupo Ciencia y Tecnología de Biomoléculas de Interés Agroindustrial (CYTBIA). Ingeniera Agroindustrial. Popayán, Colombia. https://orcid.org/0000-0002-2448-608X

4 Universidad del Cauca, Facultad de Ciencias Agrarias, Grupo Ciencia y Tecnología de Biomoléculas de Interés Agroindustrial (CYTBIA). M.Sc. Ingeniería. Popayán, Colombia. https://orcid.org/0000-0002-7899-854X

Correspondencia: silvioandres@gmail.com 